\title{
Design of Mechatronic System for a Two-Axis Inertially Stabilized Platform in an APLI System
}

\author{
Xiangyang Zhou \\ Beihang University \\ School of Instrumentation Science and electrical \\ Engineering \\ Beijing, China \\ e-mail: xyzhou@buaa.edu.cn \\ Hongyan Zhang \\ Beihang University \\ School of Instrumentation Science and electrical \\ Engineering \\ Beijing, China \\ e-mail: zhanghongyan5319@163.com
}

\author{
Beilei Zhao \\ Beihang University \\ School of Instrumentation Science and electrical \\ Engineering \\ Beijing, China \\ e-mail: zhaobeilei1992@buaa.edu.cn
}

\begin{abstract}
A two-axis inertially stabilized platform (ISP) with multi-sensors is designed which is used in unmanned helicopter (UH)-based airborne power line inspection (APLI) system. Based on the imaging requirements of remote sensors, an optimized mechanical system with high ratio of support load and self-weight is proposed. Meanwhile, a high precision control method is also designed. The performances of ISP including both of mechanical and control systems are tested by experiments. The results verify that the proposed design project is effective that attained the control accuracy and stability required by APLI system.
\end{abstract}

Keywords-airborne power line inspection; inertially stabilized platform; structure optimization; control method

\section{INTRODUCTION}

With the development of transmission lines, the unmanned helicopter (UH) for airborne power line inspection (APLI) can be better than the manual power line inspection. In an UH-based APLI system, two-axis inertial stabilized platform (ISP) is one of crucial components in an aerial remote sensing system, which is used to hold and control the line of sight (LOS) of the imaging sensors keeping relative inertial space stable [1]. The control performance will directly influence the images quality. In order to overcome the influence of the flight attitude swing and other disturbance on stabilization of sensor's LOS, ISP is used as a physical reference between aircraft and imaging sensors to separate the sensor's LOS from disturbances, so that LOS can keep track steady in inertial space.

An APLI system generally consists of an autono-mous helicopter, an ISP, a position and orientation system (POS) and multi-sensors, such as visible light camera, infrared camera, ultraviolet camera, laser scanner, and so on. The system can detect the faults of power transmission line intelligently. All multi-sensors including inertial measurement unit (IMU) of POS are installed in the ISP, so that the field vision of multi-sensors can be regulated to the required direction and target by ISP subjected to the output of POS [2]. In this paper, to meet the requirements of UH-based APLI system, a two-axis ISP with multisensors is designed. To validate the performance of mechatronics system, the simulations and experiments are carried out.

\section{STRUCTURE DESIGN}

\section{A. System Modeling}

Fig .1 shows the APLI system schematic diagram. Fig .2 shows three-dimensional CAD structural model of two-axis ISP. The model is a compromise result between series of conflicting requirements [3], such as small size and heavy load capacity, light weight and high stiffness, etc., which has to experience appropriate modifications to realize optimization through adjusting parameters. It can been seen that the two-axis ISP is mainly made up of five sub-systems, i.e., shaft supporting load system, gimbal structure system, control system, inertial measurement system and drive-transmission system, by which the main function of disturbances rejection could be realized in realtime.

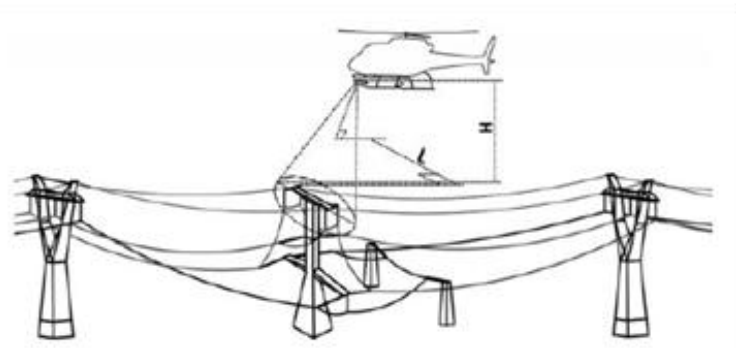

Figure 1. APLI system schematic diagram 


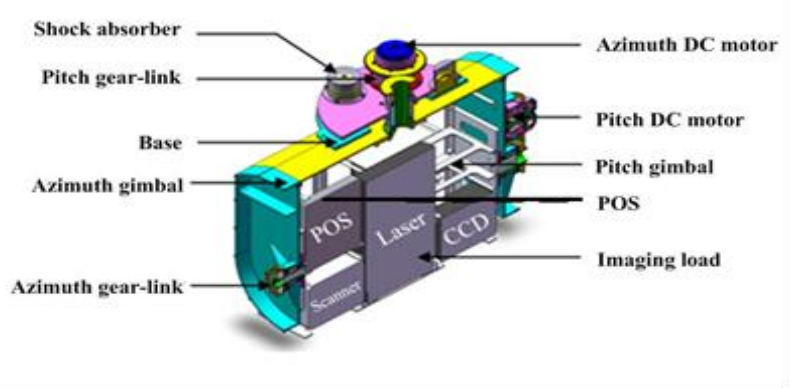

Figure 2. Three-dimensional CAD structural model of two-axis ISP

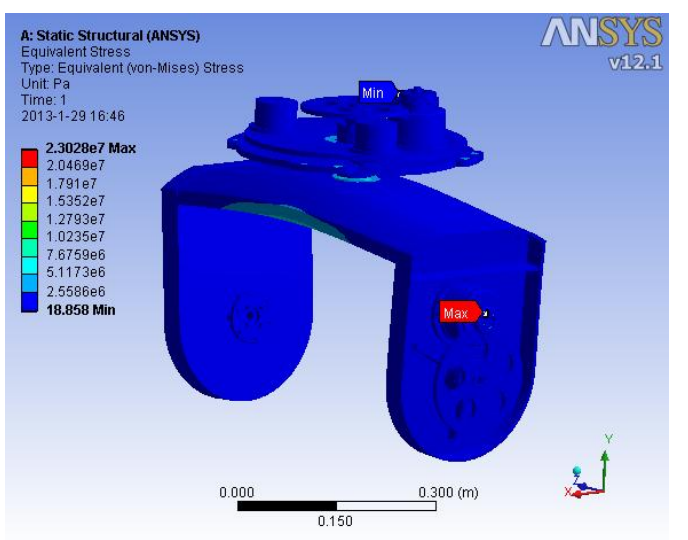

(a)

\section{B. FEM Analysis}

To validate the static and dynamic performance, finite element analysis (FEA) is performed by the ANSYS Workbench Platform (AWP). Fig .3 shows the FEA static results that satisfy the strength design requirements. Table 1 shows the natural frequencies and shapes of prior 6 modes calculated by FEA. Both of static and dynamic performance satisfies the applying requirements.

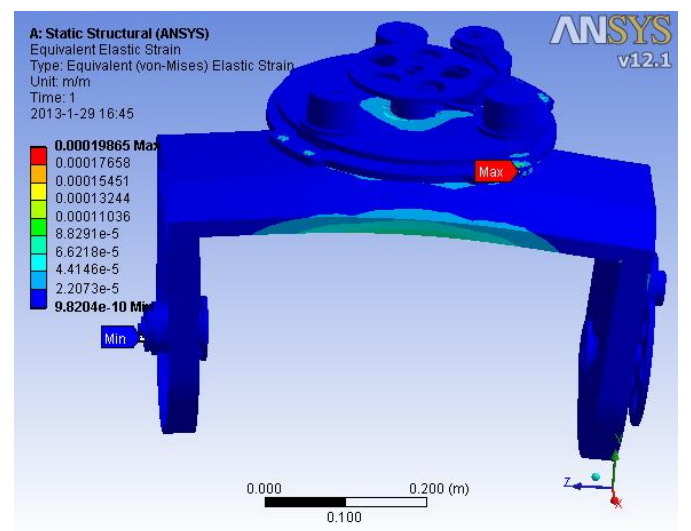

(b)

Figure 3. FEA static results: (a) Stress distribution and (b) Strain distribution.

TABLE I. THE FEA RESULTS PRIOR 6 MODES ON NATURAL FREQUENCY.

Models
$\begin{aligned} & \text { Frequency/Hz } \\ & \text { CONTROL SYSTEM DESIGN }\end{aligned}$
III.
Uses two-gimbal and two-axis structure, the
gimbal can rotate 360 ${ }^{\circ}$, and the pitch gimbal can
continuously. Imaging sensors and POS are
inside the pitch gimbal. When the imaging loads
isturbed, the control system uses the information
attitude, angular velocity, etc. to calculate the
rotational compensation torques. As a result,

Figure 4. Functional Schematic of Two-axis Stable Platform torque motor is driven to achieve the function of stabilizing imaging LOS in inertial space whose feedback is from inertial sensors [4-6]. In addition, the flight control computer provides LOS tracking command for the ISP. POS offers location and attitude information for the ISP and imaging sensors. Fig .4 shows functional schematic of two-axis stable platform.

\section{B. Control method}

A DSP-based three-loop control method is applied to the system. The current loop is made up of current sensors for armature current negative feedback to reduce the effect of source voltage fluctuations, to improve control torque linearity, to limit the motor current maximum and to protect the safety of motor and controller; the stable loop uses rate gyro as rate sensor to isolate the disturbance. The influence of disturbance to imaging loads gives back to the 
control system, to improve the response speed and stability of the system, to reduce the influence of nonlinear factors of the system, and enhance the capability of resisting disturbance; the tracking loop is main circuit of the control system, uses POS as location sensors to track the signal angle all the time. Fig .4 shows the control theory schematic of platform.

Fig .5 shows the block diagram of three-loop con-trol system for ISP. Conventional stabilization techniques employ rate gyros, rate integrating gyros, or rate sensors to detect angular rate disturbances of the LOS [2]. The blocks of G-pos, G-spe and G-cur separately represent the controllers in the position loop, speed loop and current loop; the PWM block represents the power amplification used for amplifying current to drive the torque motor; $\mathrm{L}$ represents the inductance of a torque motor and $\mathrm{R}$ represents the resistance; $\mathrm{Kt}$ represents the torque coefficient of the motor and $\mathrm{N}$ is the transition ratio from the torque motor to the gimbals; Jm represents the moment of inertia of the motor and $\mathrm{Jl}$ represents the moment of inertia of the gimbals along the rotation axis [3].

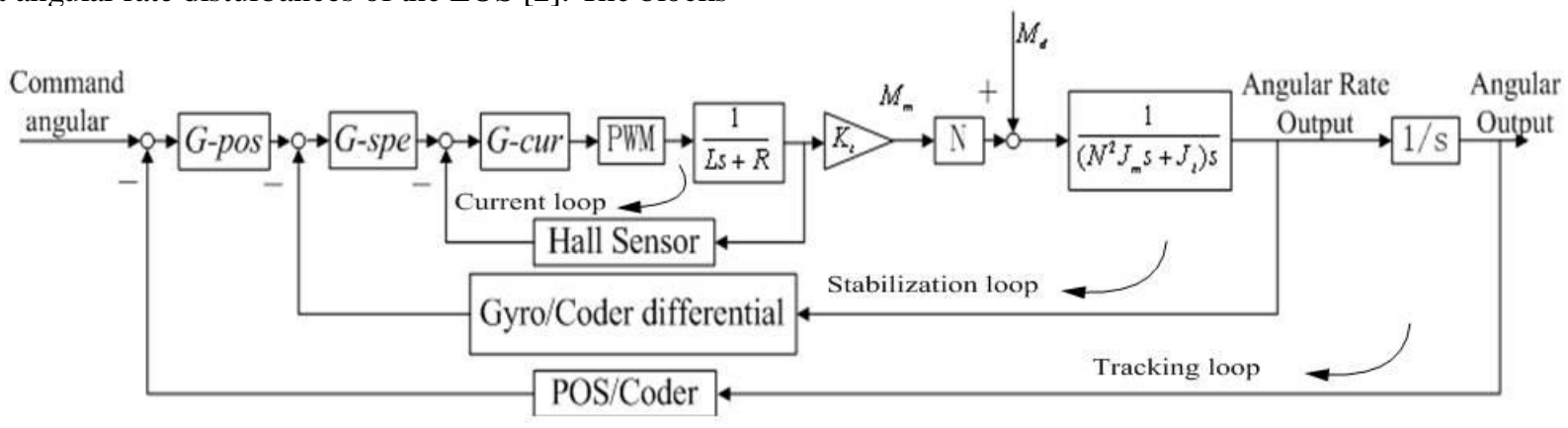

Figure 5. A block diagram of traditional three-loop control system for ISP.

\section{EXPERIMENTAL VALIDATION}

Fig . 6 shows the picture of the two-axis ISP with multisensors under the static base testing conditions. In the static testing, the two-axis ISP is fixed under the bottom of the $\mathrm{UH}$ and conducted in a stable environment. The main physical parameters of the ISP are as follows: maximum load and self-weight are $36 \mathrm{~kg}$ and $20 \mathrm{~kg}$, respectively. The range of azimuth rotation angle is $0-360^{\circ}$, the maximum pitch rotation angle range is $\pm 50^{\circ}$.

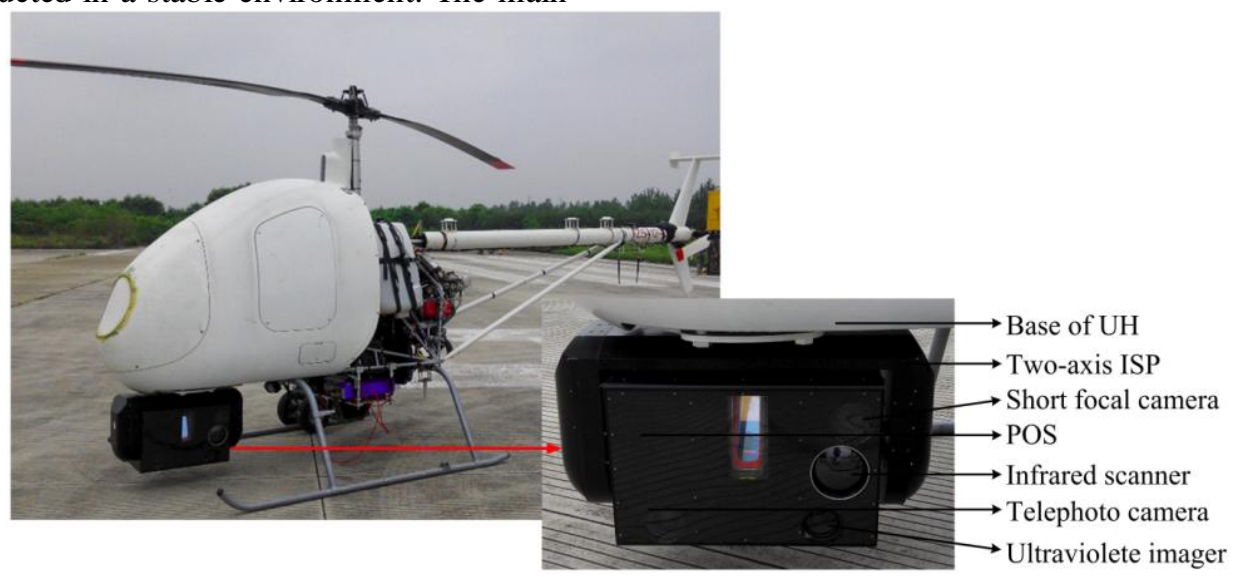

Figure 6. The picture of the two-axis ISP with multi-sensors under the static base testing conditions.

Fig .7 shows the tracking results of ISP under static base testing conditions. In experiments, the pitch and the azimuth gimbals are under the control system to follow set values. It can be seen that ISP can track steadily the instruction command with high accuracy. For pitch system, the command angles are from $-20^{\circ}$ to $-80^{\circ}$ and then reverse to $0^{\circ}$ with a short stabilization time per $20^{\circ}$ interval. For azimuth system, the stabilization performances is tested per $40^{\circ}$ interval from $180^{\circ}$ to $20^{\circ}$ and then reverse to $320^{\circ}$. 


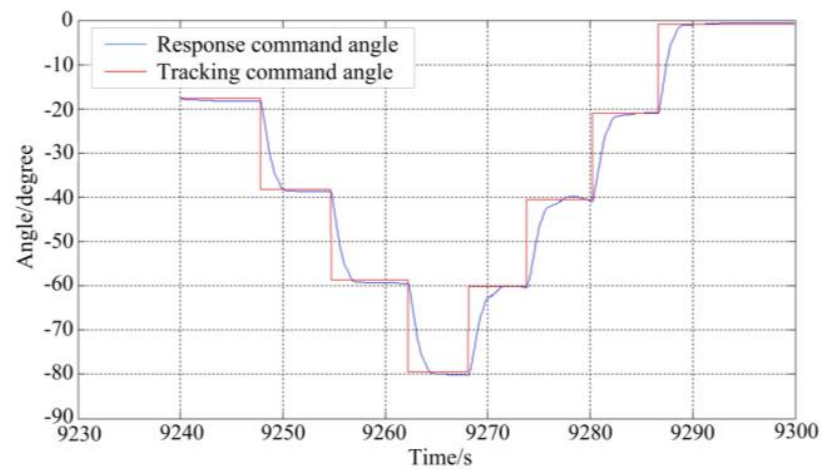

(a)

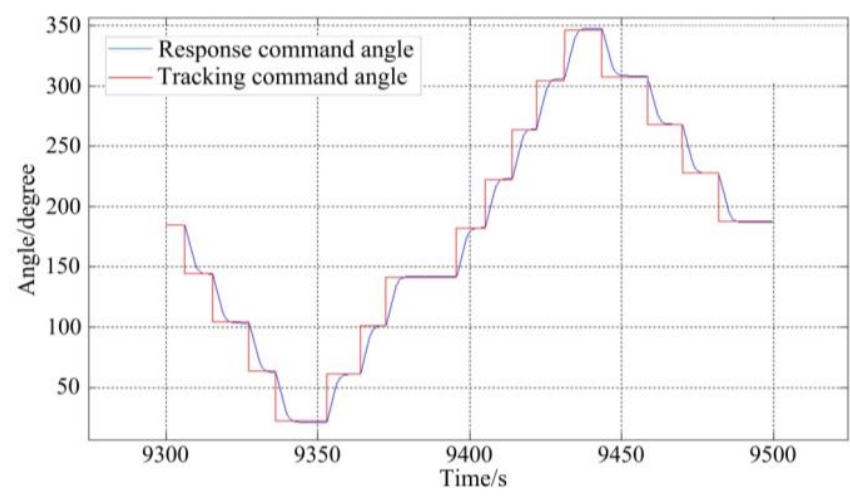

(b)

Figure 7. The tracking results of angle position under static testing: (a) the pitch system; (b) the azimuth system.

\section{CONCLUSIONS}

In this paper, a two-axis ISP for UH-based APLI system application is designed based on the multi-sensors imaging requirements. The experimental results show that the ISP system has good tracking capability to object and stabilization performance, which validate effectiveness of optimized structure and control algorithm.

\section{ACKNOWLEDGMENT}

This work was supported by the National Natural Science Foundation of China (No. 51375036 and No. 51205019).

\section{REFERENCES}

[1] Zhou Xiangyang, Yu Ruixia, Li Jianping, Li Dapeng, "Structure optimal design of roll gimbal for an aerial three-axis ISP based on FEM modal analysis," Proc. IEEE ICMTMA 2011, January 6-7, Shanghai, China, v3: 373-376.
[2] Liu, Z.C., Zhu, Z.S., Mai, X.M., Peng, X.Y., Wang, K., "Dynamic error compensation of POS in power transmission line inspection," Proc. 2013 2nd International Symposium on Instrumentation and Measurement, Sensor Network and Automation (IMSNA), Toronto, ON, Canada, 23-24 December 2013; Beijing, China, pp. 408 - 411.

[3] Zhou, X.Y., Zhao, B.L., Gong. G.H., "Control parameters optimization based on co-simulation of mechatronic system for an UA-based two-axis inertially stabilized platform," Sensors 2015, 8, 20169-20192.

[4] Ding Nan, Ding Wei, Jiang Hongxiang, "FPGA and DSP-based optical pod control system design and implementation," Navigation and control, 2013, 12 (4): 21-24.

[5] Su Pan, "Study on the control system design and filtering algorithms on gyro-stabilized pod," Harbin: Harbin Institute of Technology, 2011: 1-71.

[6] Yu Guolin, Chen Jiping, Yu Tao, Zhang Ying, Chen Xingfeng, "Rather open aviation UAV airborne remote sensing platform job control sys-tem design," Modern Electronic Technology, 2012,35 (4): 132-135. 